International Journal of Pattern Recognition and Artificial Intelligence, Vol. 10 No. 6 (1996) 749

(C) World Scientific Publishing Company

ERRATA

\title{
ON VIRTUAL PARTITIONING OF LARGE DICTIONARIES FOR CONTEXTUAL POST-PROCESSING TO IMPROVE CHARACTER RECOGNITION
}

\author{
RAINER HOCH and THOMAS KIENINGER
}

[Int. J. Pattern Recognition and Artificial Intelligence Vol. 10 No. 4 (1996) 273]

The following should appear at the end of the paper:

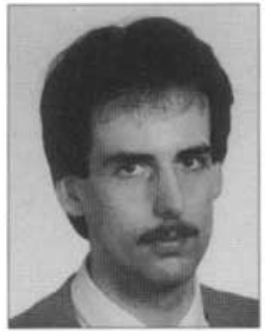

Rainer Hoch is currently a senior lecturer at the German Research Center for Artificial Intelligence (DFKI GmbH) in Kaiserslautern. His research focus has been on document analysis, in particular contextual post-processing of OCR results, with applications to information retrieval and text classification.

Dr. Hoch received his Diploma in computer science in 1989 and his Ph.D. degree in 1995 both from the University of Kaiserslautern, Germany. Before joining DFKI, he was with GMD Darmstadt where he did research in the field of electronic publishing and hypertext.

Since 1991 Dr. Hoch is a member of the document analysis group at DFKI where his main research topics focus on dictionary lookup techniques for improving OCR results and the automatic classification of documents using machine learning techniques. $\mathrm{He}$ is a member of the German GI and now responsible for several industrial projects.

Beginning March 1996, Dr. Hoch will work for SAP Inc. Germany.
Thomas Kieninger is currently working at the German Research Center for Artificial Intelligence (DFKI). Beside lexical and contextual post-processing of OCR results, his work has been in the development of a WWW based office workplace for blind people as well as the design of a user front-end to a document analysis system and a document archive. His current work aims towards layout based analysis of structural information in ASCII documents.

$\mathrm{He}$ received his Diploma in computer science from the University of Kaiserslautern, Germany, in 1994. He joined the DFKI in Kaiserslautern in 1991 as a student worker and since 1994 as a research assistant.

Photograph of T. Kieninger is not available. 